\title{
A Suprime-Cam study of the stellar population of the Ursa Major I dwarf spheroidal galaxy ${ }^{\star}$
}

\author{
S. Okamoto ${ }^{1,2}$, N. Arimoto ${ }^{2,3}$, Y. Yamada ${ }^{2}$, and M. Onodera ${ }^{4}$ \\ 1 Department of Astronomy, University of Tokyo, Hongo, Bunkyo-ku, Tokyo 113-0033, Japan \\ e-mail: sakurako.okamoto@nao.ac.jp \\ 2 National Astronomical Observatory of Japan, Osawa 2-21-1, Mitaka, Tokyo 181-8588, Japan \\ 3 The Graduate University for Advanced Studies, Osawa 2-21-1, Mitaka, Tokyo 181-8588, Japan \\ ${ }^{4}$ Institute of Earth, Atmosphere and Astronomy, BK21 Yonsei University, 134 Sinchon-dong, Seodaemun-gu, Seoul, 120-749, \\ Republic of Korea
}

Received 6 July 2007 / Accepted 4 April 2008

\section{ABSTRACT}

\begin{abstract}
We present deep and wide $V$, I CCD photometry of the Ursa Major I (UMa I) dwarf spheroidal galaxy (dSph) in the Local Group. The images of the galaxy were taken with the Subaru/Suprime-Cam wide field camera, covering a field of $34^{\prime} \times 27^{\prime}$ located at the centre of the galaxy. The colour-magnitude diagram (CMD) of the UMa I dSph shows a steep and narrow red giant branch (RGB), blue and red horizontal branch (HB), and main sequence (MS) stars. A well-defined main sequence turn-off (MSTO) is found to be located at $V_{0, \mathrm{MSTO}} \sim 23.5 \mathrm{mag}$. The distance modulus is derived as $(m-M)_{0}=19.93 \pm 0.1$ (corresponding to a distance $D=96.8 \pm 4 \mathrm{kpc}$ ) from the $V$-band magnitude of the horizontal branch $\left(V_{0, \mathrm{HB}}=20.45 \pm 0.02\right)$. The mean metallicity of the RGB stars is estimated by the $V-I$ colour as $[\mathrm{Fe} / \mathrm{H}] \sim-2.0$. The turn-off age estimated by overlaying the theoretical isochrones reveals that most of stars in the UMa I dSph are formed at a very early epoch ( 12 Gyr ago). The isopleth map of stellar number density of the UMa I dSph, based upon the resolved star counts of MS, RGB, HB stars as well as blue stragglers (BS), shows that the morphology of the UMa I dSph is quite irregular and distorted, suggesting that the galaxy is in a process of disruption. The very old and metal-poor nature of the stellar population implies that the star formation history of this newly discoverd faint $\mathrm{dSph}$ may have been different from other well-known "classical" dSphs, which show significant stellar populations of intermediate age. The stellar population of the UMa I dSph closely resembles that of Galactic old metal-poor globular clusters, but its size is typical of Galactic dSphs $\left(r_{\mathrm{e}}=188\right.$ [pc], $r_{1 / 2}=300$ [pc]), and the shape of its spatial density contours suggests that it is undergoing tidal disruption. These characteristics of stellar population and spatial distribution of the faint galaxies help us to understand how they formed and evolved, and give a hint to the nature of the building blocks of hierarchical galaxy formation.
\end{abstract}

Key words. galaxies: dwarf - galaxies: individual: Ursa Major I - galaxies: stellar content - galaxies: Local Group

\section{Introduction}

The origin of Galactic dwarf spheroidal (dSph) galaxies should be closely related to the formation and evolution history of the Milky Way. Modern cosmological models based on the Cold Dark Matter paradigm demonstrate the importance of hierarchical structure formation on all scales. Galaxies like the Milky Way and M31 form as a part of a local overdensity in the primordial matter distribution via the agglomeration of numerous smaller building blocks which independently could develop into dwarf galaxies if they escape from over take by larger ones. The relatively gas-rich dwarf irregulars still exhibit on-going star formation, while the dwarf spheroidals, being devoid of significant amounts of gas and dust, are now quiescent and are therefore, in principle, much simpler systems to study. The proximity of Galactic dSphs offers a unique opportunity for investigating galaxy formation and evolution in unprecedented detail by studying the photometric and spectroscopic properties of stellar populations.

Analyses of resolvable stellar distribution in the Sloan Digital Sky Survey (SDSS) data archive have led to recent

* Based on data collected at Subaru Telescope, which is operated by the Nationa Astronomical Observatory of Japan. discoveries of numerous very faint Galactic satellites (Willman et al. 2005; Belokurov et al. 2006; Zucker et al. 2006a,b; Belokurov et al. 2007; Irwin et al. 2007; Walsh et al. 2007). The Ursa Major I (UMa I) dwarf spheroidal galaxy located about $100 \mathrm{kpc}$ from the Milky Way is the first dSph discovered by SDSS (Willman et al. 2005). The absolute magnitude of the UMa I dSph is $M_{V} \sim-6.75$, which is about 8 times less luminous than the faintest "classical" Galactic dSphs, such as the Draco, Ursa Minor, and Sextans dSph. Measuring velocities of the UMa I stars, Kleyna et al. (2005) and Martin et al. (2007) suggested that the UMa I dSph might be the most dark matter dominated object in the Universe.

The properties of newly discovered faint $\mathrm{dSphs}$ are poorly understood. Because of their faint luminosity and size in the sky, the stellar ages, metallicities and structures of such dSphs are still unclear from previous limiting observations. The superwide field of view and enough depth of the Subaru/Suprime-Cam offers a unique opportunity for successfully and efficiently addressing the photometric aspect of this research.

This paper is organised as follows: in Sect. 2, we describe the observation and data analysis procedure. In Sect. 3, we present colour-magnitude diagrams (CMDs) of the UMa I dSph, and discuss the stellar population of the UMa I dSph. In Sect. 4, we estimate the age and metallicity of the dominant stellar population. 


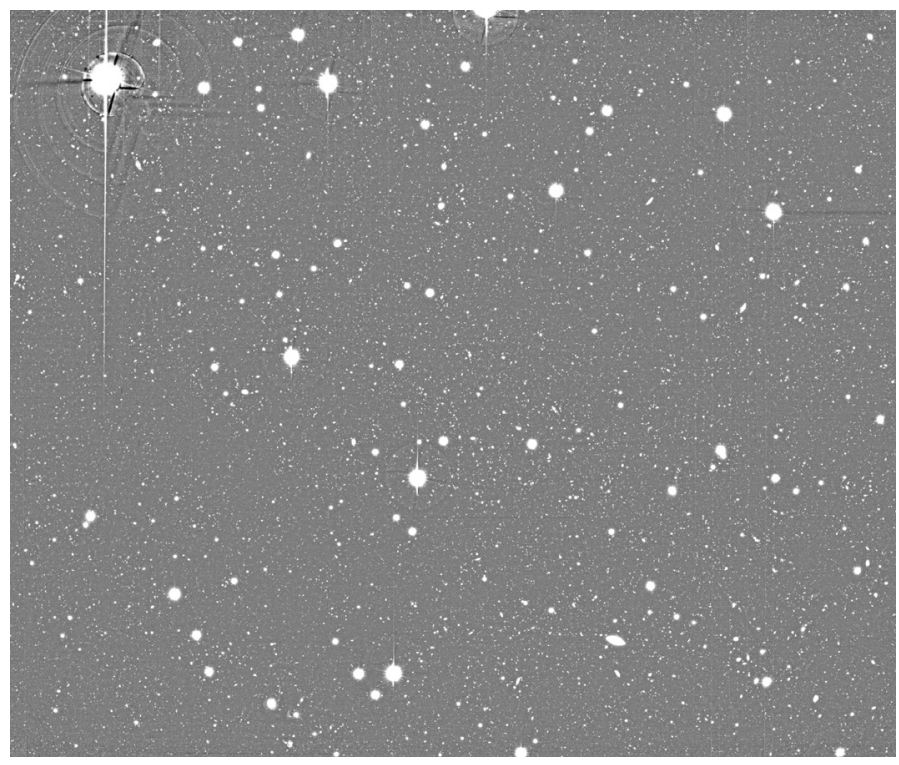

Fig. 1. The $V$-band Suprime-Cam image of Ursa Major I dSph covering a field of $34^{\prime} \times 27^{\prime}$. North is to the top and east is to the left.

In Sect. 5, we analyse the projected density profile based upon the resolved star counts. Then, we discuss the structure and evolution of the UMa I dSph and summarise this study in Sect. 6.

\section{Observation and data reduction}

We obtained deep $V, I$ CCD images of the central part of the UMa I dSph with the Suprime-Cam (Miyazaki et al. 2002) on the Subaru Telescope during the nights of 2005 December 30 to 2006 January 2 (PI. N. Arimoto). The Suprime-Cam consists of a $5 \times 2$ arrays of $2048 \times 4096$ CCD detectors and provides a field-of-view of $34^{\prime} \times 27^{\prime}$ with a pixel scale of $0.202^{\prime \prime}$. The nights of the observing run were photometric and the seeing ranged from $0.5^{\prime \prime}$ to $0.8^{\prime \prime}$. To avoid saturation of bright stars, we took long and short exposure images with Johnson $V(10 \times 50 \mathrm{~s}$ and $6 \times 10 \mathrm{~s})$ and Cousins $I(10 \times 110 \mathrm{~s}$ and $3 \times 30 \mathrm{~s})$ filters.

Data were processed using a pipeline software SDFRED dedicated to the Suprime-Cam (Yagi et al. 2002; Ouchi et al. 2004). Each image was bias-subtracted and trimmed, flat-fielded, distortion and atmospheric dispersion corrected, sky-subtracted, and combined in the usual manner. Astrometric calibration of each passband was based on a general zenithal polynomial projection derived from astrometoric standard stars selected from the online USNO catalog ${ }^{1}$. Figure 1 shows the $V$-band combined image of the UMa I dSph. Because of its faint luminosity, we cannot see the shape of the galaxy directly.

For these processed images, DAOPHOT in the IRAF package was used to obtain the point-spread-function (PSF) photometry of the resolved stars (Stetson 1987). In order to separate the point sources from the extended ones and noise-like objects, we used the DAOPHOT and $\chi^{2}$ and SHARP parameters with the following cuts: $\chi^{2}<4$ and $-0.4<$ SHARP $<0.4$. The position of detected stellar objects in each band was crosscorrelated (within $1^{\prime \prime}$ ) in order to construct catalogues of long and short exposure images. Then, combining bright stellar objects $(V<21)$ detected in short exposure images and faint stellar objects $(V>21)$ detected in long exposure images, we obtained a candidate list of about 2800 stars.

1 http://ftp.nofs.navy.mil/data/fchpix/

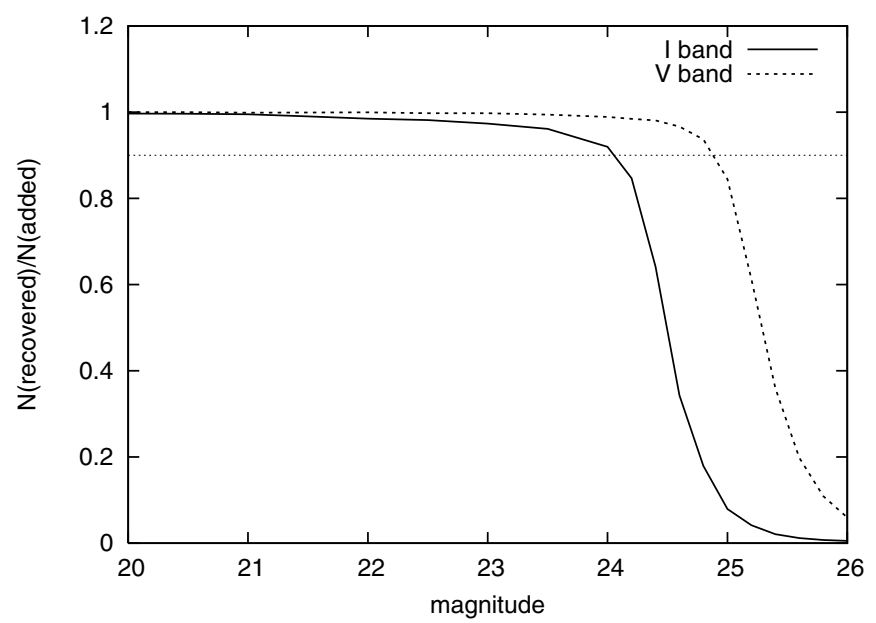

Fig. 2. Completeness of $I$ (solid line) and $V$ (doted line) photometry. The horizontal dotted line represents the $90 \%$ level.

Instrumental magnitudes of sources in the CCD images were calibrated to the standard Johnson-Cousins photometric system using the photometric standard stars of Landolt (1992), observed during the same nights. To confirm our photometric calibration, we checked the consistency of the magnitude of stars commonly detected in both long and short exposure images (typically 20-22 mag stars). The difference between the magnitude of the long and short exposures is $\Delta M_{\text {short-long }}<0.02$. The average extinction, $E(B-V)=0.019 \pm 0.005$ (corresponding to $\left.A_{V}=0.06 \pm 0.01, A_{I}=0.04 \pm 0.01^{2}\right)$ in the direction of the UMa I dSph is from Schlegel et al. (1998).

We derived completeness and photometric errors of our $V$, $I$ photometry using the artificial-star test with the ADDSTAR routine in DAOPHOT. We added 7000 artificial stars to each CCD image in each 0.5 mag interval from $18 \mathrm{mag}$ to $24 \mathrm{mag}$ and in every 0.2 mag interval from $24 \mathrm{mag}$ to $26 \mathrm{mag}$. We processed the resulting images containing artificial stars in the same way as for the original images. The detection ratio of the test, $N$ (recovered) $/ N$ (added), is plotted in Fig. 2, which shows that our photmetry is $90 \%$ complete at $V=24.9$ mag and $I=24.1 \mathrm{mag}$, respectively, independent of the position in the galaxy.

The mean errors in the photometry are based on the difference between the input magnitude and the output magnitude of the artificial stars. These errors are plotted in Fig. 3.

\section{Stellar population of the UMa I dSph}

Figure 3 show the de-reddened CMDs of the star-like objects found in the UMa I field. Error bars in both figures show the photometric errors at each magnitude level based on the artificial star test. These data are the first to reach $\sim 1$ mag below the main sequence turn-off (MSTO), and one can easily see main sequence (MS) stars of the UMa I dSph. The major features of CMDs are as follows; (i) the red giant branch (RGB) is steep and narrow. The tip of RGB is buried in the foreground stars. The intrinsic width of the RGB indicates a homogeneous chemical composition of the UMa I dSph; (ii) the horizontal branch (HB) stars are seen at $V_{0} \sim 20.45 \mathrm{mag}$; (iii) Well-defined MS stars can be traced below $V_{0} \sim 23$ mag, with the MSTO

\footnotetext{
2 The assumed extinction law is $R_{v}=3.1$ (Cardelli et al. 1989) and $A_{I} / A_{V}=0.594$ (Schlegel et al. 1998).
} 

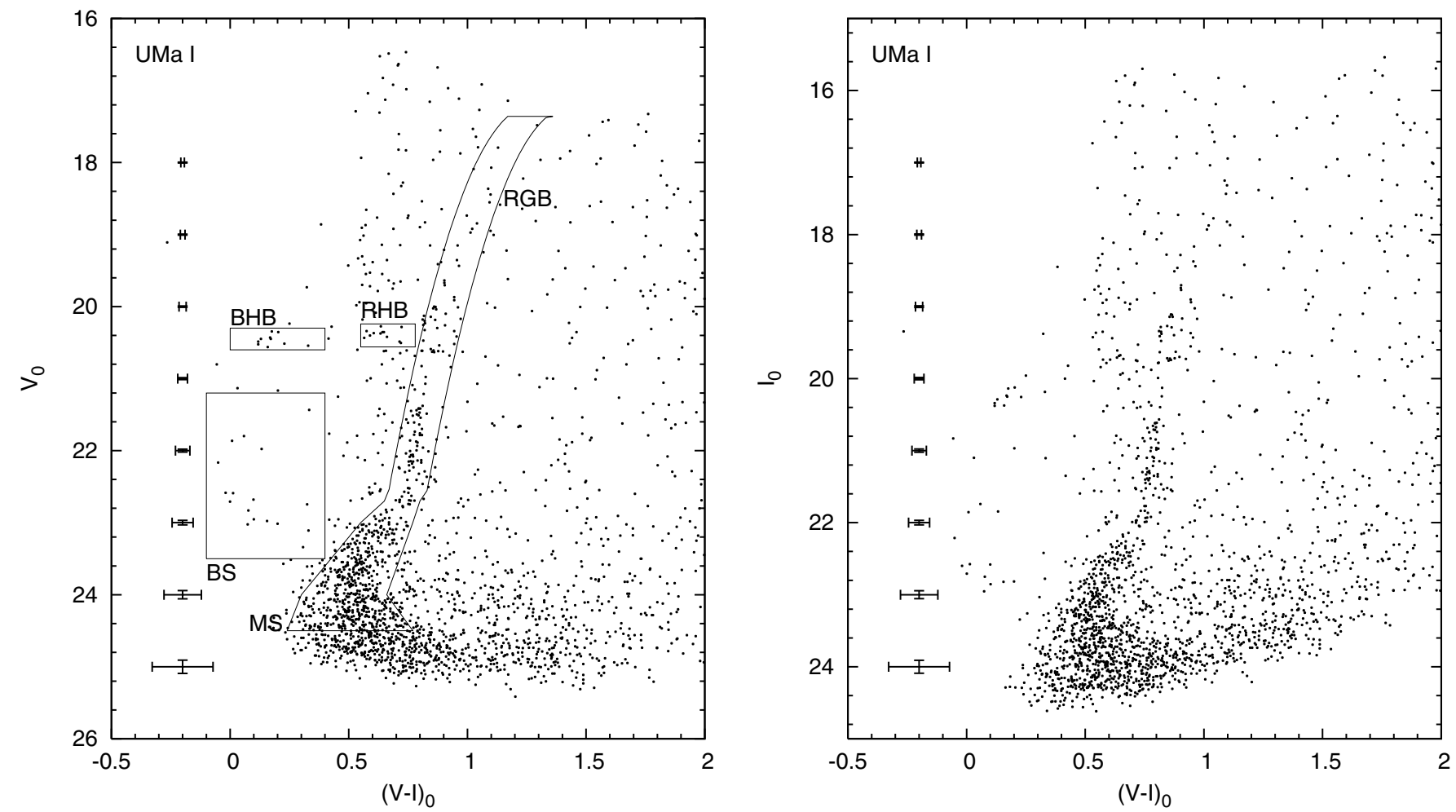

Fig. 3. Colour-magnitude diagrams of the UMa I dSph. Error bars show the photometric error at each magnitude level based on the artificial star test. The boundaries marked in the top-left panel are used to select stars belonging to the main sequence, giant branch and horizontal branch and blue strugglers of the UMa I dSph.

located at $V_{0} \sim 23.5 \mathrm{mag}$ and $(V-I)_{0} \sim 0.5 \mathrm{mag}$; (iv) there are a few blue straggler (BS) candidates. Although it has not been addressed whether these stars are young MS stars or BSs, the paucity of BS candidates in UMa I dSph suggests that the galaxy had no multiple episodes of star formation.

The CMDs are similar to those of metal-poor Galactic globular clusters, implying that the UMa I dSph has a very old and metal-poor stellar population (see Sect. 4).

These CMDs include foreground stars and background objects. The main comtamination is from the Galactic foreground stars visible at $(V-I)_{0}>0.6 \mathrm{mag}$. The contamination of the $\mathrm{RGB}$ region in the CMD could in principle be high. However, the relatively high galactic latitude of the UMa I dSph helps in reducing this effect and the MS, HB features that we consider in Fig. 3 consist predominantly of stars belonging to the UMa I dSph. In our radial profile fitting in Sect. 5, we use the TRILEGAL ${ }^{3}$ Galaxy model code (Girardi et al. 2005) to estimate the foreground star contamination.

\section{Distance, age and metallicity}

The RGB of the UMa I dSph in Fig. 3 is very thin and the tip of RGB can hardly be identified, so that the RGB tip of the UMa I dSph cannot be used as a distance indicator. We therefore have estimated the distance to the UMa I dSph using the $V$-band magnitude of the HB stars. We estimate the HB magnitude as $V_{0, \mathrm{HB}}=20.45 \pm 0.02$. Since the UMa I dSph seems to have stellar population similar to Galactic metal-poor globular clusters, we assume a theoretical value of the absolute magnitude $M_{V, \mathrm{HB}}=0.515_{-0.03}^{+0.08}$, corresponding to $[\mathrm{Fe} / \mathrm{H}]=-2.0_{-0.5}^{+0.5}$

${ }^{3}$ http://trilegal. ster . kuleuven . be/cgi-bin/trilegal
(Catelan et al. 2004), which gives the distance modulus (distance $)$ as $(m-M)_{0}=19.93 \pm 0.1(96.8 \pm 4 \mathrm{kpc})$.

In the left panel of Fig. 4, we compare the $V-(V-I)$ diagram with the fiducial sequences of metal-poor Galactic globular clusters M 3, M 5 and M 92, taken from Johnson et al. (1998); the metallicities of these clusters are $[\mathrm{Fe} / \mathrm{H}]=-1.57,-1.99,-2.24$, respectively (Harris 1996). We adopt the distance moduli to $(m-M)_{0}=14.99,14.46,14.67$, and reddening corrections to $E(B-V)=0.01,0.03,0.02$ for M 3, M 5 and M 92, respectively (Harris 1996). The mean RGB and MSTO of the UMa I dSph are similar to those of M 5 and M92, suggesting that the metallicity of the UMa I dSph is $[\mathrm{Fe} / \mathrm{H}] \simeq-2$ and the age is as old as M 5 and M92.

Our metallicity estimate is consistent with previous photometric and spectroscopic studies (Willman et al. 2005; Martin et al. 2007), and consistent with the metallicity we assumed to estimate the distance $\left([\mathrm{Fe} / \mathrm{H}]=-2.0_{-0.5}^{+0.5}\right)$.

By overlaying theoretical isochrones on the deep CMD, we estimate the age of stellar population in the UMa I dSph. The isochrones in the right panel of Fig. 4 are interpolated Padova isochrones corresponding to a metallicity $[\mathrm{Fe} / \mathrm{H}]=-2.0$ and age of 10.0, 12.6, 14.1 Gyr (Girardi et al. 2002). These isochrones are made by the interpolation with the Padova isochrones of metallicity $Z=0.0001$ and $Z=0.0004$. The right panel of Fig. 4 shows that the fiducial sequence of MSTO of the UMa I dSph is best reproduced by the isochrone of $12.6 \mathrm{Gyr}$, consistent with the age roughly estimated by the comparison with Galactic old metal-poor globular clusters in the left panel. Consequently, most of stars in the UMa I dSph are estimated to have formed at least 12 Gyr ago.

The UMa I dSph has an old metal-poor stellar population with a narrow dispersion of age and metallicity. This 

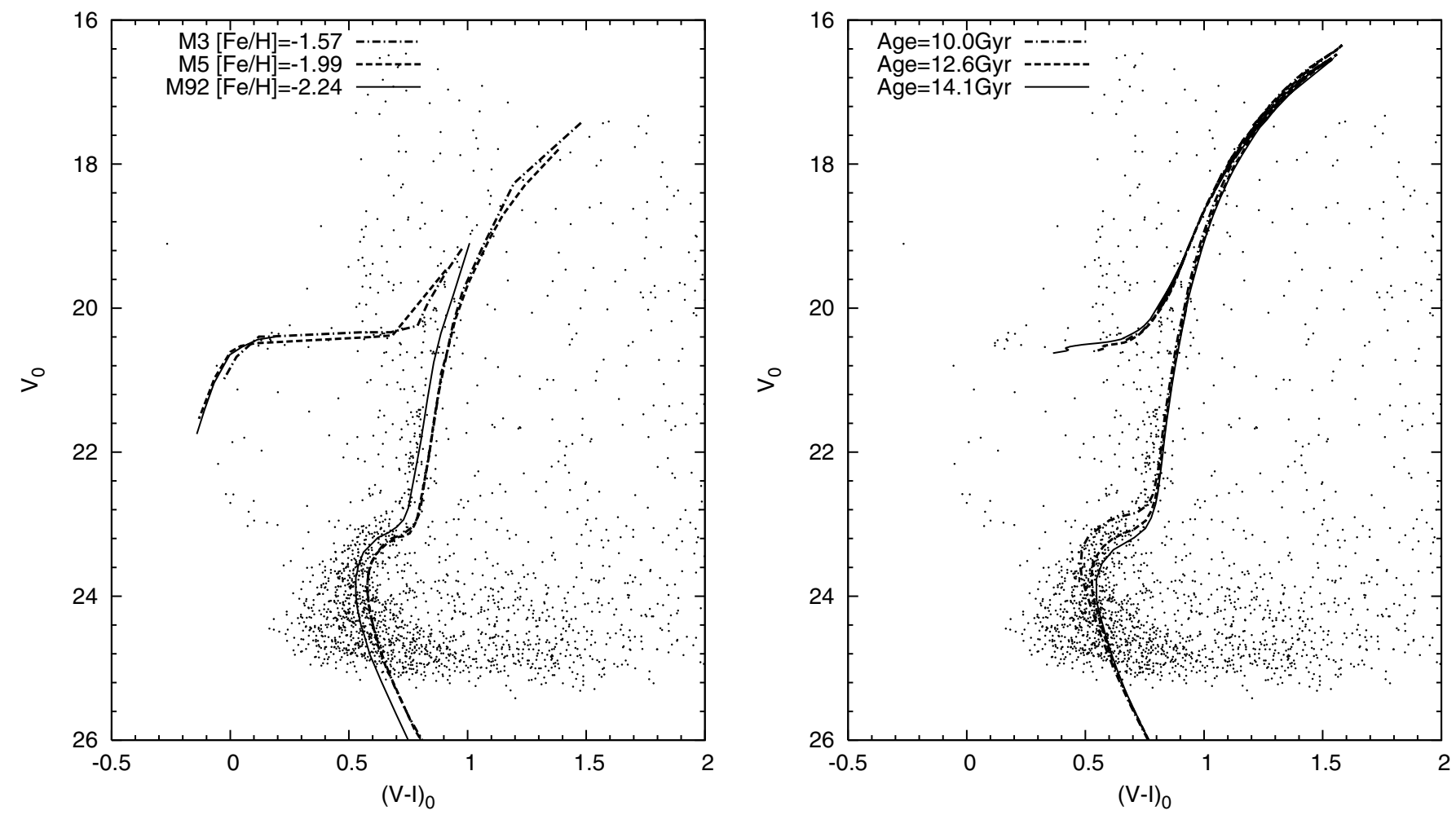

Fig. 4. Left: $V$-band colour-magnitude diagram of the UMa I dSph with the fiducial sequence of Galactic globular clusters (M3, M 5, M 92). Right: the theoretical isochrones overlaid in the $V$-band colour-magnitude diagram of the UMa I dSph. The lines stand for the interpolated Padova isochrones for $[\mathrm{Fe} / \mathrm{H}]=-2.0$ and ages of 10.0, 12.6, 14.1 Gyr which were shifted according to the distance of the UMa I dSph.

characteristic of the stellar population of the UMa I dSph seems to be different from "classical" dSphs, which show evidence of multiple stellar populations of various ages and metallicities (e.g. Shetrone et al. 2001; Ikuta \& Arimoto 2002; Tolstoy et al. 2004). The UMa I dSph is well-described by a single stellar population, such as a typical globular cluster.

\section{Structural properties of the UMa I dSph}

Even though its stellar population closely resembles that of Galactic old metal-poor globular clusters, the spatial distribution of the UMa I dSph shows different properties in morphology and size. Figure 5 shows the spatial distribution and the density contour map of stellar sources, that were selected as candidate stars of MS, RGB, HB and BS from the CMD shown in Fig. 3 using the selection boxes marked. These stars are binned in $30^{\prime \prime} \times 30^{\prime \prime}$ boxes and smoothed by a Gaussian kernel of bandwidth $=0.6^{\prime}$ in the lower panel of Fig. 5. The plotted contour levels are $1-10 \sigma$ above the background level. The isodensity contours covering the central $730 \mathrm{pc} \times 670 \mathrm{pc}$ of the UMa I dSph have quite irregular shapes, stretched toward the upper left and lower right on Fig. 5. This corresponds to the tangential direction toward the Galactic centre.

We also compare the spatial distributions of subgiant stars $(23.0<V<23.4)$ and faint MS stars $(24.0<V<24.4)$ to study the mass segregation in the UMa I dSph, but there is no difference in both stars.

To calculate the structural parameters, we use the candidate members in Fig. 3 to derive the centroid from the densityweighted first moment of their spatial distribution, and average ellipticity and position angle using the three density-weighted second moments (e.g. Stobie 1980). The radial profile shown in Fig. 6 is derived by calculating the average number density within elliptical annuli. The number density of foreground stars in the direction of the UMa I dSph is estimated as $0.05 \mathrm{arcmin}^{-2}$ by using TRILEGAL (Girardi et al. 2005). We fit the radial profile with standard exponential and Plummer models, and estimate the half-light radius $\left(r_{1 / 2}\right)$ of both models as $11.30^{\prime} \pm 0.50^{\prime}$ and $10.42^{\prime} \pm 0.76^{\prime}$, respectively. Hereafter the radius is the elliptical radius $r=\left[x^{2}+y^{2} /(1-e)^{2}\right]^{1 / 2}$, where $e$ is the ellipticity of the galaxy, $x$ and $y$ is the coordinate aligned with the major and minor axis, respectively. The estimated half-light radius is larger than the previous estimation of $r_{1 / 2} \sim 7.75^{\prime}$ (Willman et al. 2005), probably due to our much deeper images. The field level of Willman I (Willman et al. 2006) which lies within a few degrees of UMa I is within the error bars of the field in our profile.

The half-light radius of the UMa I dSph corresponds to $300 \mathrm{pc}$, which is an order of magnitude larger than that of the largest Galactic globular clusters. The total luminosity of the candidate members is used to estimate the lower limit of the central surface brightness as $\mu_{0, V}=29.5 \pm 0.2\left[\mathrm{mag} / \mathrm{arcsec}^{2}\right]$. These best-fitting structural parameters are listed in Table 1.

The structural parameters allow us to constrain the dark matter content of the UMa I dSph, assuming that it is in virial equilibrium. The mass-to-light ratio of a simple stellar system with a symmetric distribution can be estimated as $M / L=$ $9 \eta \sigma^{2} / 2 \pi G r_{\mathrm{hb}} \Sigma_{0}$, where $\eta$ is a dimensionless parameter dependent on the luminosity distribution, $\sigma$ is the central velocity dispersion, $r_{\mathrm{hb}}$ is the half-light radius, and $\Sigma_{0}$ is the central surface brightness (Richstone \& Tremaine 1986). Using the velocity dispersion values $\sigma=9.3\left[\mathrm{~km} \mathrm{~s}^{-1}\right]$ (Kleyna et al. 2005) and $\sigma=11.9\left[\mathrm{~km} \mathrm{~s}^{-1}\right]$ (Martin et al. 2007), the mass-to- $V$ band light ratio of the $\mathrm{UMa} \mathrm{I} \mathrm{dSph}$ is $M / L \sim 1500$ and $2500 M_{\odot} / L_{\odot}$, 
(pc)

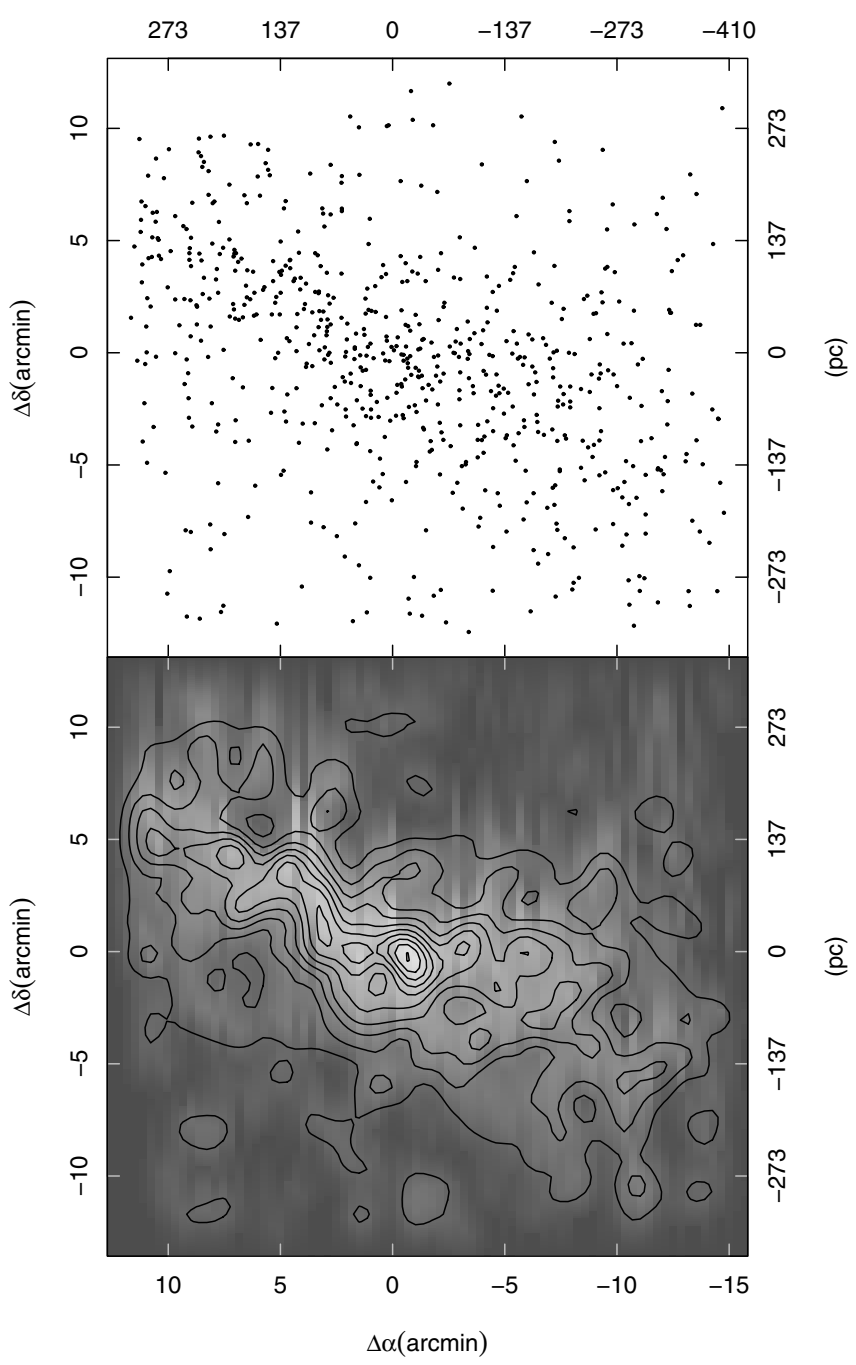

Fig. 5. Upper: the spatial distribution of the stars selected by the dashed lines in Fig. 3, which cover the central $730 \mathrm{pc} \times 670 \mathrm{pc}$ of the UMa I dSph. Lower: the isodensity contour of the CMD-selected sources. The plotted contour levels are $1-10 \sigma$ above the background level.

respectively. This estimate implies that the UMa I dSph is a highly dark matter dominated galaxy. However, the shape of the spatial density contours suggests that the UMa I dSph is undergoing tidal disruption. If this is the case, the galaxy may not be in virial equilibrium.

\section{Discussion and summary}

We have used Subaru/Suprime-Cam to obtain multi-colour imaging of the UMa I dSph which is sensitive enough to derive the stellar population below the MSTO and wide enough to study the spatial distribution of stars in the galaxy. Our deep and wide photometry allows us to study more accurately than previous study. The distance modulus of the UMa I is derived as $(m-M)_{0}=19.93 \pm 0.1$, corresponding to $96.8 \pm 4 \mathrm{kpc}$. We identify a number of stars in several different evolution phases, MS, RGB, HB and BS, which are characterized as a metal-poor single old population. The mean metallicity is estimated by the $V-I$ color of RGB stars to $[\mathrm{Fe} / \mathrm{H}] \sim-2.0$, and the turn-off age

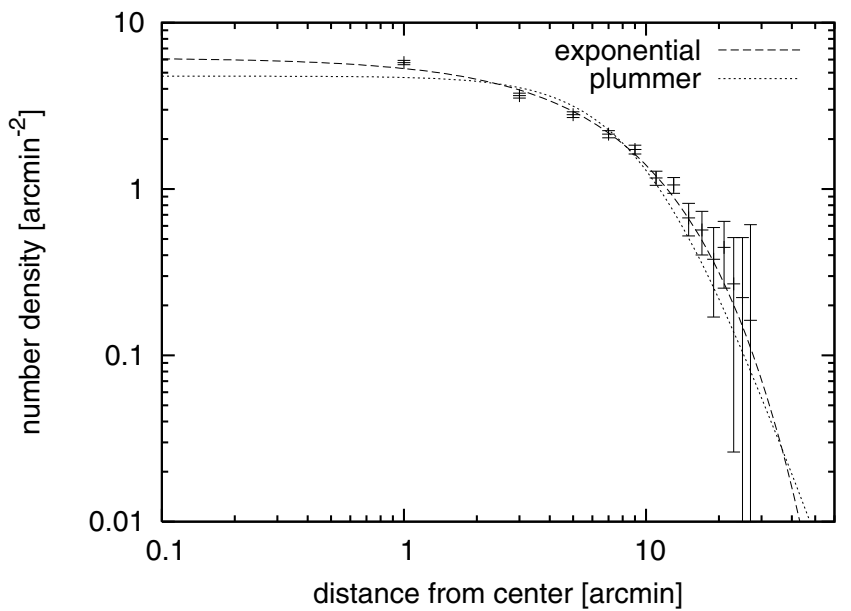

Fig. 6. Radial profile derived by calculating the average number density within elliptical annuli. The effect of foreground stars is corrected by using a Galactic model (Girardi et al. 2005). The best-fitting exponential profile with the effective radius $r_{\mathrm{e}}=6.73^{\prime} \pm 0.30^{\prime}$ and Plummer profile with the Plummer radius $b=10.42^{\prime} \pm 0.76^{\prime}$ have been overlaid as the dashed line and dotted line, respectively.

Table 1. Structural parameters of Ursa Major I dSph.

\begin{tabular}{lc}
\hline \hline Parameter & Value \\
\hline Coordinates $(\mathrm{J} 2000)$ & $10^{\mathrm{h}} 34^{\mathrm{m}} 44.4^{\mathrm{s}},+51^{\circ} 55^{\prime} 33.9^{\prime \prime}$ \\
Position angle & $78^{\circ} .12$ \\
Ellipticity & 0.54 \\
$r_{1 / 2}$ (exponential) & $11.30 \pm 0.50[\mathrm{arcmin}]$ \\
$r_{1 / 2}$ (Plummer) & $10.42 \pm 0.76[\mathrm{arcmin}]$ \\
$\mu_{0, V}$ (exponential) & $29.5 \pm 0.2\left[\mathrm{mag} / \mathrm{arcsec}^{2}\right]$ \\
$\mu_{0, V}$ (Plummer) & $29.7 \pm 0.4\left[\mathrm{mag} / \operatorname{arcsec}^{2}\right]$ \\
$(m-M)_{0}$ & $19.93 \pm 0.1$ \\
Distance & $96.8 \pm 4[\mathrm{kpc}]$ \\
\hline
\end{tabular}

reveals that the most of the stars in the UMa I dSph were formed at least 12 Gyr ago.

Most "classical" Galactic dSphs have signs of multiple stellar populations - different spatial distributions of red and blue HB stars (e.g. Harbeck et al. 2001; Tolstoy et al. 2004). The $[\alpha / \mathrm{Fe}]$ ratio of most stars so far observed in the dSphs is lower than for Galactic halo stars of similar metallicity (Shetrone et al. 2001, 2003), which implies that star formation in such dSphs lasted for several Gyr (Ikuta \& Arimoto 2002). On the other hand, the UMa I dSph seems to have a single metal-poor old population, and some of the other newly discovered dSphs also show signs of a similar stellar population (Bootes I: Belokurov et al. 2006; Coma Berenices: Belokurov et al. 2007; Hercules: Coleman et al. 2007) and appear to have a different stellar population than well-known "classical" dSphs. Why are these dSphs so faint and have a single old population? If the $\mathrm{SN}$-driven winds blow the gas earlier in such a low-mass galaxy because of the relatively shallow potential, the stellar population may retain the information of the initial star formation activity of the galaxy. The abundance patterns of these galaxies allow us to reveal the nature of the galaxy evolution.

Although the CMD of the UMa I dSph shows a single epoch of star formation as a metal-poor globular cluster, its spatial size is typical of Galactic dSphs $\left(r_{1 / 2}=300\right.$ [pc] $)$, and the shape suggests that it is undergoing tidal disruption. The morphology of UMa I dSph is quite irregular, stretched and distorted. A similar 
feature is found in the Hercules $\mathrm{dSph}$ which shows an extremely flat structure $(e \sim 0.65)$ (Coleman et al. 2007). If the tidal force could cause such an elongated shape, these galaxies would have had high orbital ellipticities and underwent tidal disruption at the last pericenter passage to the Milky Way.

With the usual assumptions of symmetry and virial equilibrium, the UMa I dSph seems to have high $\mathrm{M} / \mathrm{L}$ and would be a dark matter dominated object. If so, the UMa I dSph is presumed to be a long-lived object with distorted morphology. It is unclear whether the UMa I dSph has been caught in the act of dissolving into the Galactic halo, or has maintained such an elongated shape dominated by dark matter for a long time. The high-dispersion spectroscopic data will necessary to further study the chemical and dynamical evolution of this galaxy.

Acknowledgements. We wish to express our gratitude to the anonymous referee for very helpful suggestions and comments. The authors are thank to T. Kodama, Y. Komiyama, L. Greggio and A. Renzini for suggestions. We also thank the observatory staff of the Subaru Telescope. S.O. special thanks to M. Iye for support and comment. This work is supported by a Grant-in-Aid for Science Research (No. 19540245) by the Japanese Ministry of Education, Culture, Sports, Science and Technology.

\section{References}

Belokurov, V., Zucker, D. B., Evans, N. W., et al. 2006, ApJ, 647, L111 Belokurov, V., Zucker, D. B., Evans, N. W., et al. 2007, ApJ, 654, 897
Cardelli, J. A., Clayton, G. C., \& Mathis, J. S. 1989, ApJ, 345, 245 Catelan, M., Pritzl, B. J., \& Smith, H. A. 2004, ApJ, 154, 633 Coleman, M. G., De Jong, J. T. A., Martin, N. F., et al. 2007, ApJ, 668, L43 Girardi, L., Bertelli, G., Bressan, A., et al. 2002, A\&A, 391, 195

Girardi, L., Groenewegen, M., Hatziminaoglou, E., \& da Costa, L. 2005, A\&A, 436,895

Harbeck, D., Grebel, E. K., Holtzman, J., et al. 2001, AJ, 122, 3092

Harris, W. E. 1996, AJ, 112, 1487

Ikuta, C., \& Arimoto, N. 2002, A\&A, 391, 55

Irwin, M. J., Belokurov, V., Evans, N. W., et al. 2007, ApJ, 656, 13 Johnson, J. A., \& Bolte, M. 1998 AJ, 115, 693

Kleyna, J. T., Wilkinson, M. I., Evans, N. W., \& Gilmore, G. 2005, ApJ, 630, L141

Landolt, A. 1992, AJ, 104, 340,

Martin, N. F., Ibata, R. A., Chapman, S. C., Irwin, M., \& Lewis, G. F. 2007, MNRAS, 380, 281

Miyazaki, S., Komiyama, Y., Sekiguchi, M., et al. 2002, PASJ, 54, 833

Ouchi, M., Shimasaku, K., Okamura, S., et al. 2004, ApJ, 611, 660

Richstone, D. O., \& Tremaine, S. 1986, AJ, 92, 72

Schlegel, D. J., Finkbeiner, D. P., \& Davis, M. 1998, ApJ, 500, 525

Shetrone, M. D., Côté, P., \& Sargent, W. L. W. 2001, ApJ, 548, 592

Shetrone, M. D., Shetrone, V. K. A., Tolstoy, E., et al. 2003, AJ, 125, 684

Stetson, P. B., PASP, 99, 191

Stobie, R. S. 1980, J. British Interplanet. Soc., 33, 323

Tolstoy, E., Irwin, M. J., Helmi, A., et al. 2004, ApJ, 617, 119

Walsh, S. M., Jerjen, H., \& Willman, B. 2007, ApJ, 662, L83

Willman, B., Dalcanton, J. J., Martinez-Delgado, D., et al. 2005, ApJ, 626, L85

Willman, B., Masjedi, M., Hogg, D. W., et al. 2006 [arXiv: astro-ph/0603486]

Yagi, M., Kashikawa, N., Sekiguchi, M., et al. 2002, AJ, 123, 66

Zucker, D. B., Belokurov, V., Evans, N. W., et al. 2006a, ApJ, 643, L103

Zucker, D. B., Belokurov, V., Evans, N. W., et al. 2006b, ApJ, 650, L41 